\title{
Seeding Element Polarity Arrangement on Drum-Type Magnetic Precision Seeder
}

\author{
Qirui Wang*, Jianping Hu, Qi Liu, and Junchao Yan \\ Key Laboratory of Modern Agricultural Equipment and Technology, \\ Ministry of Education \& Jiangsu Province, Jiangsu University, Zhenjiang,212013, China, \\ Tel.: +86-15862938529 \\ wangqirui2@126.com
}

\begin{abstract}
In order to improve Multi-polar magnetic structure's efficiency. Ansoft Maxwell of electromagnetic field finite element software is utilized to study the effect of different polarity arrangement of magnetic seeding element on the magnetic induction intensity of seeding element end and magnetic field distribution in the seeding air gap. As a result of the different electromagnetic coil current direction, magnetic polarity side is different, space structure of magnetic field distribution is also different. The suction metering device of the scope and efficiency is decided by the magnetic field distribution. According to the results of finite element analysis of magnetic field, research on magnetic drum seed-metering device with the design of the program components, and enhance the precision seed metering device performance. Studies have shown that the magnetic induction intensity of seeder in the same polarity arrangement such as NN or SS is lower than in the arrangement of cross with NS, and magnetic field is uniform along the circumference and non- uniform along the axial in the both the arrangement.
\end{abstract}

Keywords: Magnetic seeding element, Polarity arrangement, Magnetic induction analysis.

\section{Introduction}

Precision sowing that is a modern seed technology, is general one seed to one plug hole. At present, at home and abroad, advanced precision seeder mainly use airsuction and mechanical way. Such as Shuttle international companies of Holland's needle precision seeder. Features of air-suction precision sowing is that seed size not strict, higher planting accuracy, but the structural complexity, manufacturing costs are high. Mechanical structure is simple precision seeding, planting speed, but seed size is strict. For shortcomings of precision seeder, in this paper, the new principle of magnetic-type seed is raised.

Magnetic system is consisted of many magnetic seeding elements which are arranged by the form of 4 rows uniform along the circumference and the axial layout by way of distance of Plug hole. As shown in Figure 1, The principles of its absorbing

\footnotetext{
* Corresponding author.
} 
seeds is that the seeds coated magnetic material comes with the magnetic seeding element to do circular motion, when the magnetic components are passing the seedbox. For different particle size of the seeds, just change the solenoid current, precision seed can be realized. As a result of the direction of current is difference, the polarity of the seeding elements is different. Space composed of magnetic field distribution (K J Binns,B Sc and so on, 1997) is different and space distribution of magnetic field directly determines the metering device performance. In the following study the effects of the magnetic field of space with the different magnetic order (YANG and CHEN, 2009).

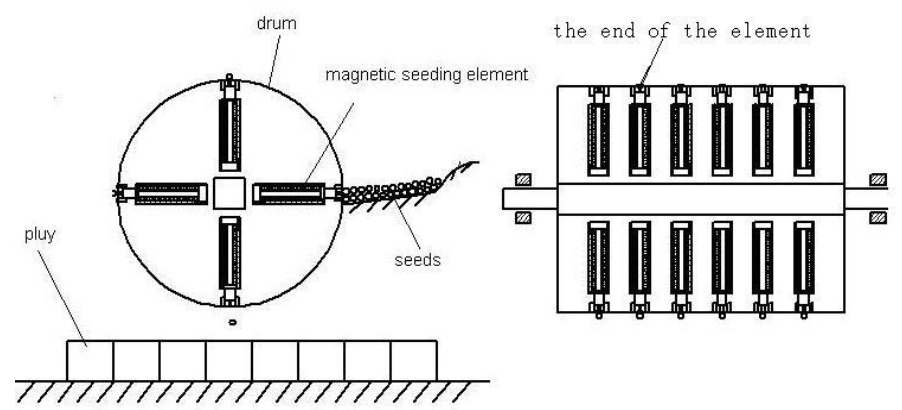

Fig. 1. Schematic diagram of Precision Seeder

\section{The Study of Structure of the Magnetic Polarity}

Effect of spatial distribution of magnetic field of Polarity is manly about magnetic circuit which is generated between magnetic components $\mathrm{N}, \mathrm{S}$ pole. There are two main aspects, which the components of seed-polar components effect the space magnetic field. Two aspects are along the circumferential direction and the radial direction. Following we through the finite element analysis software (Pearson J, Squire P T, Maylin M G, et al. 2000), to study the magnetic field with different polarity and the spatial distribution.

\subsection{Effect of Polarity Order of the Magnetic Seeding Elements with Circumferential Direction}

There are two means that is NN magnetic order and NS magnetic order, about distribution of the direction of the polar circle (Dawson F P, Hideo OKA,1991). As shown in Figure 2, the following we analysis of different polarity on the case with the magnetic circuit, taking the condition of the 400 coil turns and current, the distribution of the magnetic line of force as shown in Figure 2.

As figure 2 the polarity in different circumstances stations, magnetic circuit has changed dramatically, when we used NN magnetic order, most of the magnetic line of force through composed of the covers from the core and the magnetic circuit. A small number of magnetic lines pass through magnetic circuit between two ends of the core. When used NS magnetic order, most of the magnetic line of force through composed 


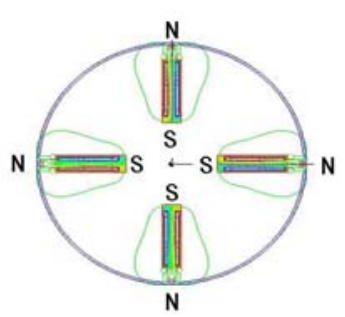

(a) NN same polarity arrangement

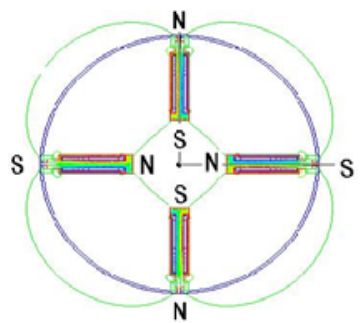

(b) NS different polarity arrangement

Fig. 2. Distributing of magnetic force line under two magnetic polar order

of the covers from the core and the magnetic circuit, The magnetic line of force losing in space along in the circumferential direction of seed magnetic circuit consisting of components. It can be seen the magnetic seeding elements along the circumferential direction have mutual effect. When used different magnetic orders magnetic seeding elements are interaction, and the space corresponding magnetic field changes.

Through the distribution of magnetic line of force with different polarity, magnetic interact between the components. The effect of specific size is the change in magnetic induction. Magnetic induction distribution along the circumference show in figure 3 and 4. Abscissa for the circle's circumference, unite is $\mathrm{mm}$. Longitudinal coordinates for the magnetic induction intensity, unite is T. There are four seeding magnetic elements along circumferential direction .In figure 3, 4 raised at the two ends of a single convex, there are four convex. The convex position is position of seeding magnetic element's end face. As the figure 3 and 4, magnetic induction intensity is relatively uniform, when there four seeding magnetic elements along circumference. When used $\mathrm{NN}$ magnetic order, the face value of seeding magnetic elements is $0.058 \mathrm{~T}$. When used NS magnetic order, the face value of seeding magnetic elements is 0.060T. the face value of seeding magnetic elements change $2 \mathrm{mT}$. Along the circumferential direction the polarity order of the magnetic seed components have a certain interaction. Therefore we used NS magnetic order along the circumferential direction.

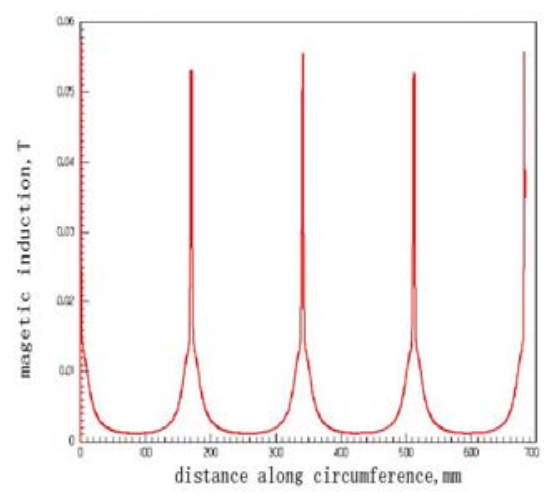

Fig. 3. The curve of magnetic induction intensity in the order of $\mathrm{NN}$ along the circumferential direction

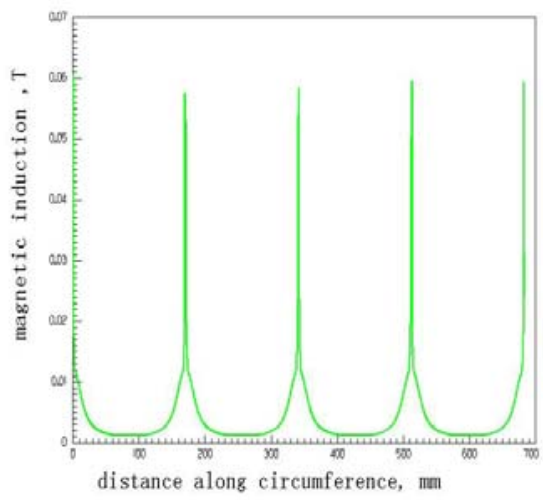

Fig. 4. The curve of magnetic induction intensity in the order of NS along the circumferential direction 


\subsection{Effect of Polarity Order of the Magnetic Seeding Elements Along Axial Direction}

The effect of polarity of the seeding magnetic elements along axial direction is the effect between the seeding magnetic elements in a row. There are six seeding magnetic elements in arrow. Now study the magnetic field diversification with differentpolarity. There are also two means that is NN magnetic order and NS magnetic order. As shown in Figure 5,6.

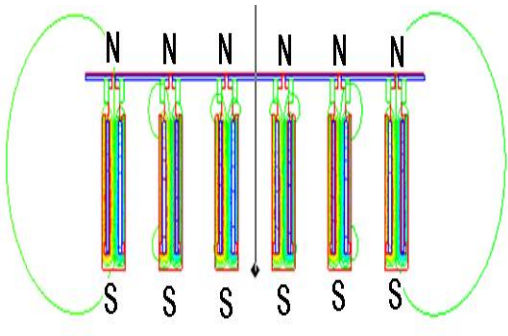

Fig. 5. NN order of space distribution of magnetic field lines of magnetic force

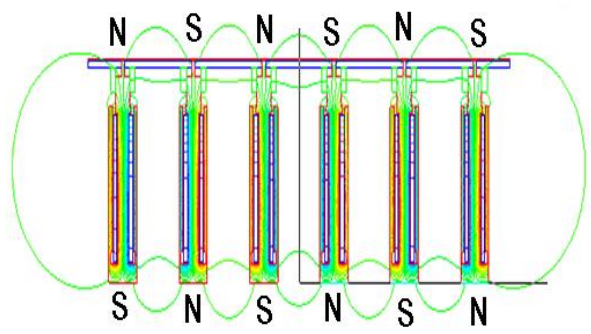

Fig. 6. NS order of space distribution of magnetic field lines of magnetic force

The following we analysis of different polarity on the case with the magnetic circuit, taking the condition of the 400 coil turns and current, the distribution of the magnetic line of force as shown in Figure 5 and 6. When we used NN magnetic order, as result of the air magnetic resistance, when adjacent seeding magnetic elements arrange with same polarity .Magnetic lines of force pass through the core and magnetic shield and form magnetic circuit. There is no magnetic circuit (POWELL J D , ZIEL INSKI A E, 1999 ) between the magnetic elements. When we used NS magnetic order, most of the space magnetic field lines are passing through the magnetic circuit between the core and the magnetic shield. As a result of adjacent magnetic polarity opposite, some losing magnetic force lines in the air through the magnetic circuit which is constituted by adjacent magnetic polarity. Thus to know: the effect between magnetic elements which in a row, is obvious.

Along the cylinder wall seeding magnetic elements of end-point draw a horizontal line, study magnetic field changes in the line. The length of roller is 240 millimeters. Abscissa is length of a roller and ordinate is magnetic induction. Magnetic induction intensity changes along a the line as figure 7 and 8.As figure 7 When we use NN magnetic order, the magnetic induction of the middle seeding magnetic elements is relatively smaller, the size is $0.02 \mathrm{~T}$. But the magnetic induction intensity on sides is relatively larger, about $0.029 \mathrm{~T}$.

The distribution of the magnetic field distribute as strong - weak - strong form. When the adjacent magnetic polarity is the same, they have effect of weaken each other magnetic field. As figure 8, when we used NS magnetic order, the magnetic induction of the middle seeding magnetic elements is relatively bigger, the most size is $0.070 \mathrm{~T}$. 


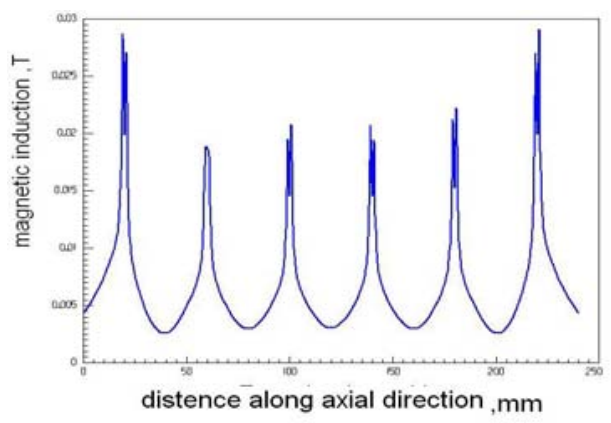

Fig. 7. The curve of magnetic induction intensity in the order of $\mathrm{NN}$ along the axial direction

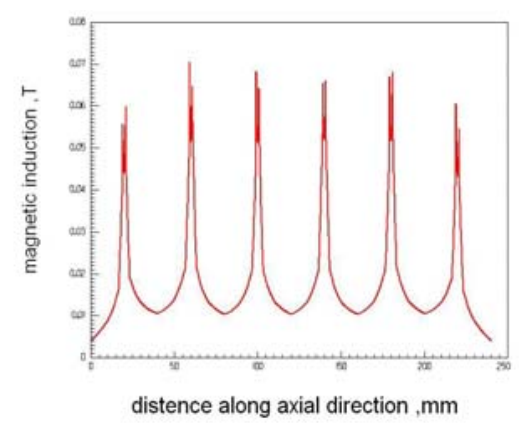

Fig. 8. the curve of magnetic induction intensity in the order of NS along the axial direction

Table 1. Magnetic induction intensity of the seeding element end (T)

\begin{tabular}{crllllll}
\hline & \multicolumn{6}{c}{ The number of magnetic seeding element } \\
\cline { 3 - 8 } & Type & 1 & 2 & 3 & 4 & 5 & 6 \\
\multirow{2}{*}{ NN } & Measured(Bi) & $2.89 \mathrm{E}-2$ & $1.92 \mathrm{E}-2$ & $2.21 \mathrm{E}-2$ & $2.28 \mathrm{E}-2$ & $2.42 \mathrm{E}-2$ & $2.98 \mathrm{E}-2$ \\
& Bi-B min & $0.97 \mathrm{E}-2$ & 0 & $0.29 \mathrm{E}-2$ & $0.36 \mathrm{E}-2$ & $0.50 \mathrm{E}-2$ & $1.06 \mathrm{E}-2$ \\
\hline \multirow{2}{*}{ NS } & Measured(Bi) & $6.02 \mathrm{E}-2$ & $7.37 \mathrm{E}-2$ & $7.22 \mathrm{E}-2$ & $6.98 \mathrm{E}-2$ & $7.20 \mathrm{E}-2$ & $6.10 \mathrm{E}-2$ \\
& Bi-B min & 0 & $1.35 \mathrm{E}-2$ & $1.20 \mathrm{E}-2$ & $0.96 \mathrm{E}-2$ & $1.18 \mathrm{E}-2$ & $0.08 \mathrm{E}-2$ \\
\hline \multicolumn{2}{c}{$\mathrm{Bi}(\mathrm{NS})-\mathrm{Bi}(\mathrm{NN})$} & $3.13 \mathrm{E}-2$ & $5.45 \mathrm{E}-2$ & $5.01 \mathrm{E}-2$ & $4.70 \mathrm{E}-2$ & $4.78 \mathrm{E}-2$ & $3.12 \mathrm{E}-2$ \\
\hline
\end{tabular}

When the magnetic induction intensity on sides is relatively smaller, about 0.055T. The distribution of the magnetic field distribute as weak-strong - weak form. When the adjacent magnetic polarity is opposite, they have effect of weaken each other magnetic field. They have effect of enhance each other's magnetic field. As table 1 the magnetic induction intensity of six elements of difference polarity arrangement, we can know the most size of induction of seeding magnetic elements from 0.0298T to $0.0737 \mathrm{~T}$, Increase $147.32 \%$. The least size of induction of seeding magnetic elements is from $0.0192 \mathrm{~T}$ to $0.0602 \mathrm{~T}$, Increase $213.54 \%$. The changing of magnetic induction of the end face of seeding magnetic elements is obvious.

\section{Conclusions}

(1) Adopting different polarity order along the circumferential direction, the magnetic induction intensity of the seeding elements end did not change significantly.

(2) Adopting the NN same magnetic order along the axial direction, the distribution of the magnetic field distribute as strong - weak - strong form; when we use NS magnetic order, the distribution of the magnetic field distribute as weak - strongweak form. 
(3) Adopting the same or different polarity order along the axial direction, the magnetic induction intensity of the seeding elements end had great difference. When the magnetic seeding elements were arranged across with the NS polarity order along the axial direction, its magnetic induction intensity at the end of the seeding elements was bigger than arrangement with the $\mathrm{NN}$ same polarity order.

(4) According to the research results, we suggest adopting a sequence of magnetic NS and increasing Coil number of magnetic seeding elements of two side of the drum.

\section{Acknowledgements}

This work was financially supported by the open fund of Jiangsu Provincial Key Laboratory of Modern Agricultural Equipment and Technology, Jiangsu University (NZ200604), by Eleventh Five-Year-Plan National Scientific \& Technological Supporting Project (2006BAD11A10) and Nature Science Fund of Jiangsu province (BK2007088).

\section{References}

Binns, K.J., et al.: Compution treedimensional finiteelement solution of permanent magnet machines. IEEE Trans. on MAG17 (6), 2997-2999 (1997)

Yang, M.-z., Chen, H.: On Electromagnetic Fields Flux Density. Journal of Shanxi Institute of Education. IEEE. Trans. Magnetics 33(1), 3255-3258 (2009)

Pearson, J., Squire, P.T., Maylin, M.G., et al.: Biaxial stress effects on the magnetic properties of pure iron. IEEE. Trans. Magnetics 36(1), 3251-3253 (2000)

Dawson, F.P., Hideo, O.: Flux Control Model of A Ferrite Orthogonal Core. IEEE Tran. Magnetics 27(6), 5259-5261 (1991)

Powell, J.D., Ziel Inski, A.E.: Observation and si2 mulation of solid2armature railgun performance. IEEE Trans. on Magn. 35(1), 84289 (1999)

Powell, J.D., Ziel Inski, A.E.: Current and heat transport in the solid2armature railgun. IEEE Trans. on Magn. 31(1), 6452650 (1995) 